\title{
Virtual-reality-based educational laboratories in fiber optic engineering
}

Dana Hayes, Craig Turczynski, Jonny Rice, Michael Kozhevnikov

Dana Hayes, Craig Turczynski, Jonny Rice, Michael Kozhevnikov, "Virtualreality-based educational laboratories in fiber optic engineering," Proc. SPIE 9289, 12th Education and Training in Optics and Photonics Conference, 928921 (17 July 2014); doi: 10.1117/12.2070779

SPIE Event: 12th Education and Training in Optics and Photonics Conference, 2013, Porto, Portugal 


\title{
Virtual-Reality-Based Educational Laboratories in Fiber Optic Engineering

\author{
Dana Hayes, Craig Turczynski, Jonny Rice and Michael Kozhevnikov* \\ Department of Engineering, Norfolk State University, VA, USA 23504
} \\ *mkozhevnikov@nsu.edu
}

\begin{abstract}
Researchers and educators have observed great potential in virtual reality (VR) technology as an educational tool due to its ability to engage and spark interest in students, thus providing them with a deeper form of knowledge about a subject. The focus of this project is to develop an interactive VR educational module, Laser Diode Characteristics and Coupling to Fibers, to integrate into a fiber optics laboratory course. The developed module features a virtual laboratory populated with realistic models of optical devices in which students can set up and perform an optical experiment dealing with laser diode characteristics and fiber coupling. The module contains three increasingly complex levels for students to navigate through, with a short built-in quiz after each level to measure the student's understanding of the subject. Seventeen undergraduate students learned fiber coupling concepts using the designed computer simulation in a nonimmersive desktop virtual environment (VE) condition. The analysis of students' responses on the updated pre- and post tests show statistically significant improvement of the scores for the post-test as compared to the pre-test. In addition, the students' survey responses suggest that they found the module very useful and engaging. The conducted study clearly demonstrated the feasibility of the proposed instructional technology for engineering education, where both the model of instruction and the enabling technology are equally important, in providing a better learning environment to improve students' conceptual understanding as compared to other instructional approaches.
\end{abstract}

Keywords: virtual reality, fiber optics, optical coupling, computer simulation

\section{INTRODUCTION}

Virtual reality (VR) technology has made great strides in the last two decades. The advancement of speed and power of both CPUs and graphics cards were key in the general advancement of VR technology by improving the quality and complexity of virtual environments (VEs), while demand for such technology (mostly by the gaming community) has reduced cost. Additionally, immersive visualization hardware such as head-mounted displays has become cheaper, more ergonomic, and better performing. Many applications for VR technology have been explored, including vehicle training simulations, computer-aided design, data visualization (ex., seismological data for oil companies), psychiatric treatment, and entertainment. ${ }^{1}$ VR technology has gained the interest of researchers studying human factors and perception because of its potential to provide an interactive, realistic environment for study that can be precisely controlled and easily manipulated. These qualities make a VE ideal for producing consistent, reproducible results in these types of studies. ${ }^{2}$

Researchers and educators have observed great potential in VR technology as an educational tool due to its ability to engage and spark interest in students, thus providing them with a deeper form of knowledge about a subject. To date, there are a number of academic institutions and companies exploring and promoting the use of VR-based simulations for science education, with more than 100 reported research efforts and a couple dozen practical commercialized tools in a variety of fields. The rich, immersive, and interactive VEs made possible by VR simulation and graphics technology can be used to help students understand various scientific concepts. When it comes to mastering abstract scientific concepts, it helps if a student is able to create, or is provided with, a basic mental model - usually involving invisible factors that represent intangible forces and other abstractions - of what they believe is going on $^{3}$; this is an area in which an engaging VR simulation could prove very useful. An interactive computer or web-based program can also help students put what they have learned in lecture into practice taking their learning from memorization to tacit learning (learning from taking action, such as a laboratory course), a method that can enable students to acquire skills and become leaders in the workplace. $^{4.5}$

Some of the research on VR technology has focused on studying the effectiveness of 3D and interactive simulations as classroom tools. Simulations can be helpful when it is difficult or impractical to either perform an experiment (due either to lack of equipment, complexity, or cost) or to observe its inner workings (ex. the propagation of light through the

12th Education and Training in Optics and Photonics Conference, edited by

Manuel F. P. C. Martins Costa, Mourad Zghal, Proc. of SPIE Vol. 9289, 928921

(C) 2014 SPIE, OSA, IEEE, ICO · doi: 10.1117/12.2070779

Proc. of SPIE Vol. $9289928921-1$ 
atmosphere). ${ }^{6}$ VR simulations have three characteristics that make them useful in research and education: autonomy (the ability of events within the world to occur on their own), presence (the user's immersion into the world), and interaction (the user's ability to affect the world). Thus, a student could become deeply involved in a virtual world and learn the effects of the different changes he/she could impose on it. The interaction aspect of VR is of particular interest because a user can easily view objects from different perspectives and alter variables that cannot be altered in nature. ${ }^{7}$

Figure 1 shows the ideal relationship between a real experiment and a VR simulation of that experiment. A virtual lab could provide an easily accessible middle step between the theories taught in class and the real experiment. A simulation can present subjects to students in an easy-to-understand way, while providing enough realism (relative to real-world mechanics) that the student could verify what he/she has seen in class and in the real lab by doing the virtual lab, and vice-versa.

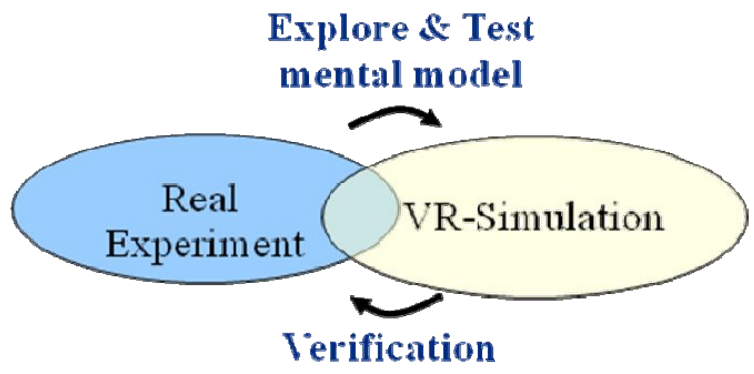

Figure 1. Connection between real experiments and VR simulations.

Specifically for simulations in optics and photonics, VR technology currently exists mostly in the form of professional and scientific design and analysis tools and imply a full grasp of optical theory on the part of the user. ${ }^{8-10}$ As such, they assume that the user has a high level of knowledge in the theories, concepts, and methods present within these programs and are not ideal for classroom use. Also, they are neither designed to allow students to learn about a system nor do they measure a student's progress through the program. A curriculum-based interactive educational program would be more desirable as a tool for instruction; this would reinforce specific concepts and theories taught in the classroom in an engaging, interactive setting to help students gain a stronger grasp of optics and accommodate students with different learning styles in order to benefit as many students as possible. In order to be the most effective, this program must be able to present subjects in an educational context-i.e., a subject would be broken down and explained so that the student could fully understand the principles presented.

The goal of this project is to develop a prototype of an interactive, educational module in Fiber Optics that will be integrated into a laboratory course. The developed module features a virtual laboratory populated with realistic models of optical devices in which students can set up and perform an optical experiment dealing with laser diode characteristics and fiber coupling.

\section{METHODOLOGY}

\subsection{Participants and activities}

Seventeen undergraduate students learned fiber coupling concepts using the designed computer simulation in a nonimmersive desktop VE condition. All of the students were tested individually. First, the participants were pre-tested with a Diagnostic Problem Solving Questionnaire (DPSQ), which measures qualitative and quantitative conceptual understanding of optical fiber coupling. Potential questions were gathered from a variety of sources, including homework assignments from other university's engineering courses. The DPSQ test consisted of N=12 items and the internal reliability (alpha Cronbach) of the test is 0.5. After the pre-test, all participants were exposed to a virtual simulation on optical coupling (the module features are described in the next section). Students used the module individually, making their way through each level with a guide containing exercises they could perform while within a particular level. The activities in the fiber optic simulations in general lasted for 45-50 min. After completing these activities, all of the participants were post-tested on the same DPSQ they were administered on the pre-test. Finally, all of the participants were given a concluding questionnaire, in which they were asked to describe which aspects of the 
simulation were particularly helpful for understanding optical coupling, as well as to address any difficulties with the simulation and technical problems.

\subsection{Design and Development of the VR Module Prototype}

This module is designed to teach the student about fiber coupling to a semiconductor diode source. The module has an experimental setup where light coming from a diode gets focused by a biconvex lens in order to couple into a fiber. Throughout the program the student learns about two different kinds of coupling loss; loss due to mismatched area and loss due to mismatched numerical aperture (NA) - as well as the effects of different parameters of the optical system on these losses.

The prototype is being designed with Vizard, a Python-based, 3D virtual environment programming software developed by WorldViz, LLC (Santa Barbara, CA). The program features an environment that mimics the real-world optics labs at Norfolk State University (NSU). The optical system presented within this lab consists of a virtual lab room with an optical table at its center (see Figure 2). On this table sit a laser diode driver (LDD), temperature controller unit (TEC), laser diode, biconvex lens, and a short length of fiber (complete with visible core and cladding). The diode, lens, and fiber are "mounted" to the table via posts and post holders. The room is sized to give a sense of size and space to the simulation; it is possible to "walk" around the entire optical table within the room.



Figure 2. Screenshot of the first level of the module.

The designs for the optical table, LDD, TEC, posts, post holders, and diode were based on real-life Newport-brand equipment that can be found in NSU's optics labs. In fact, the textures on the LDD, TEC, and optical table were created from photos taken of their real-life counterparts. Originally we intended to have the diode be controlled through the virtual LDD and TEC units, but as we added more parameters for the students to control (including factors that cannot be changed in real life, such as the diode's emitting angle) we felt that it made more sense to have the student control everything from a menu. Instead, the LDD and TEC sit on the optical table as decorations since they are commonplace in NSU's optics labs.

The development was subject to interactive refinement and modifications guided by experimentation and evaluation. This task also included a customization of existing graphical user interface of Vizard 3.0 to add windows and menus vital for the proposed courseware implementation. Custom graphical interface developed directly for the proposed learning activity gives the product a more cutting edge form and functionality. 
The program calculates the coupling efficiency of the system as well as the output power of the system. The set of equations used to calculate these values have been programmed into Vizard. The results of these calculations are presented with a "scoreboard" displaying the coupling efficiency as well as real-time graphs of both the coupling efficiency and optical power versus time. Laser light within this system is represented by semi-transparent cones; one coming from the face of the diode representing the initial laser light and another coming from the lens representing the focused light. A third cone, coming from the fiber's face, represents the fiber's NA or acceptance cone.

The developed simulation allows (a) High flexibility in parameter settings, (b) Real time graphing capabilities (e.g., coupling efficiency as a function of time), (c) Additional visualization tools (e.g., the emission cone of the diode or the acceptance cone of the fiber).

The main module parameters and features are summarized in Table 1.

Table 1. Module features

\begin{tabular}{|c|c|c|}
\hline \multicolumn{2}{|c|}{ Program Features } & $\begin{array}{l}\text { - Virtual 3D optics lab, } \\
\text { - Models of laser diode, biconvex lens, and optical fiber, } \\
\text { - Light cones to represent light from laser, light refracted by lens, } \\
\text { fiber acceptance cone, } \\
\text { - Assessment quizzes between each level, } \\
\text { - Recording of student’s quiz responses. }\end{array}$ \\
\hline \multirow{4}{*}{ 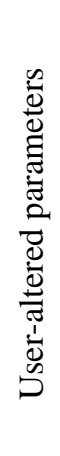 } & Distance & $\begin{array}{l}\text { - Distance between laser and lens, } \\
\text { - Distance between lens and fiber. }\end{array}$ \\
\hline & Laser diode & $\begin{array}{l}\text { - Power, } \\
\text { - Emission angle, } \\
\text { - Emitting surface (laser size). }\end{array}$ \\
\hline & Lens & - Focal length \\
\hline & Fiber & $\begin{array}{l}\text { - Diameter, } \\
\text { - Numerical aperture. }\end{array}$ \\
\hline
\end{tabular}

\subsection{VR Module Flow}

The virtual learning module has three levels of increasing complexity with assessment quizzes occurring after each level (see Figure 3). The object of each level is to adjust different parameters in order to achieve $100 \%$ coupling efficiency within the system, with each level giving the student control over a different set of the system's parameters.

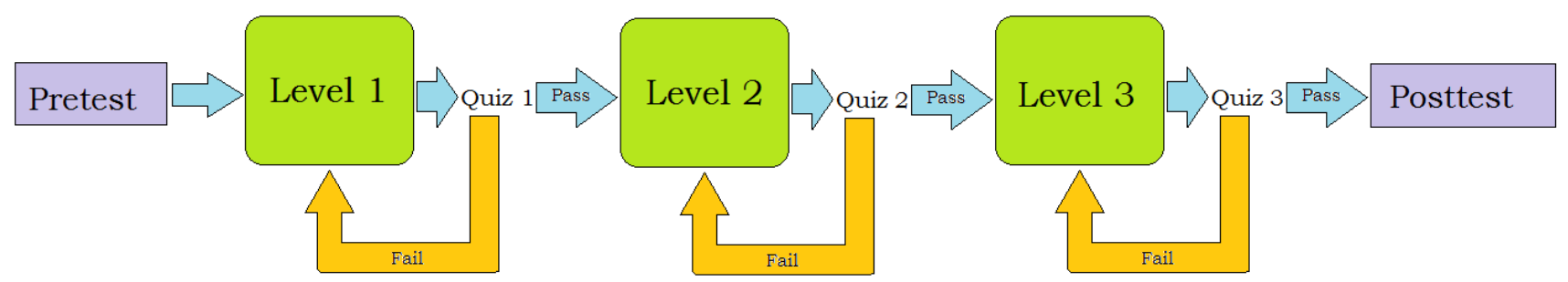

Figure 3. Schematic of the module's level and quiz structure.

A short user's manual was developed in order to help guide students through the module. The manual includes descriptions of the program's level system, built-in quizzes, navigation system, graphs, and menus. Several screenshots 
from the module are included to illustrate the look and features of the module. A lab procedure accompanies this manual. This document gives students a set of exercises they can perform within each level (in addition to their own exploring). The procedure also gives directions to students who miss a quiz question, either suggesting they repeat an exercise or complete an additional one, so that they may discover the correct answer.

The first level deals with coupling loss due to mismatched area between the laser spot size, lens diameter, beam size after the lens, and fiber diameter. The second level deals with coupling loss due to mismatched NA between the laser, lens, and fiber. The third stage deals with coupling loss due to all factors. This level structure is accomplished by disabling some of the parameters in the first two levels so that the student can focus on the type of loss presented. In the third level, all parameters are available, and the student has the maximum allowed control over the system.

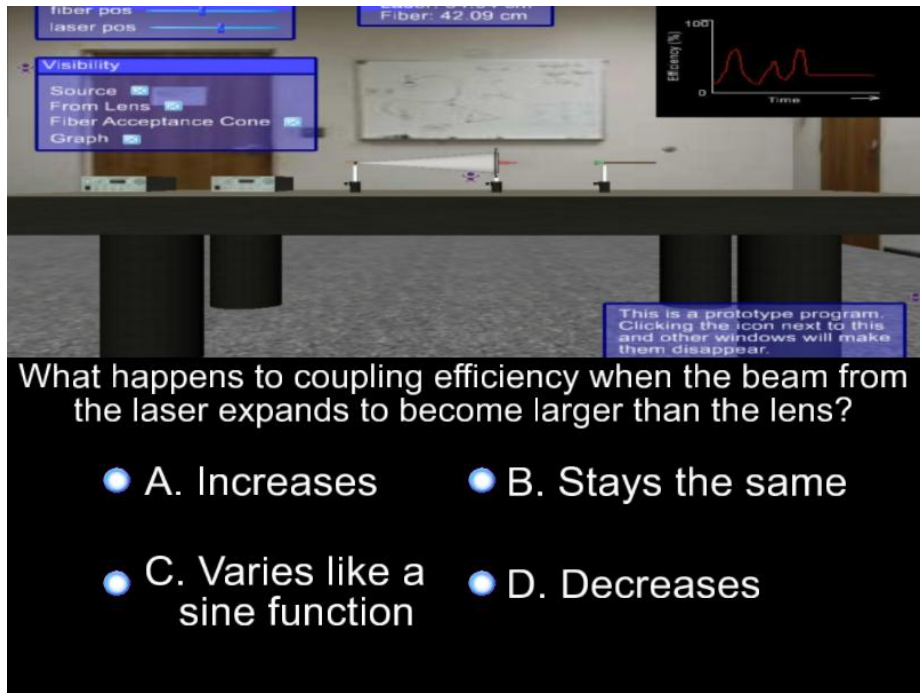

Figure 4. Screenshot of a built-in quiz.

When a student decides to move on from a level of the program he/she simply clicks on a button located at the bottom of the screen in order to take the quiz for that level. This short, built-in assessment quiz (see Figure 4), contains one or two questions on each level, that the student must pass in order to advance to the next level or finish the virtual lab (the program records and saves all quiz responses to a text file on the host computer). Answering any of the questions incorrectly will send the student back to the previous level where students can further "play" with the module in order to discover the right answer. The questions are based on the intended learning outcome of that particular level. If the student fails the quiz he/she will have to redo the level and will be provided hints (instructions are given in the lab procedure) to help him/her figure out the right answer. All quiz responses are recorded in a text file in order to track the student's progress and can be retrieved and viewed by a professor/evaluator.

\section{RESULTS}

The analysis of students' responses on the updated pre- and post test show statistically significant improvement of the scores for the post-test as compared to the pre-test. While on the pre-test the proportion of correctly solved questions was 0.45 ( $\mathrm{SD}=0.15)$, on the post test, the proportion of correctly solved questions was $0.56(\mathrm{SD}=0.15)$. Paired-samples t-test revealed that this improvement was statistically significant: $\mathrm{t}(12)=-3.386, \mathrm{p}=0.005$.

On the evaluation questionnaire, there were six questions asking students to rate particular aspects of the developed module on a rating scale which ranged from 1 to 6 . The students gave high ratings for the items that asked them to rate the helpfulness of this software in understanding optical coupling concepts (mean score $=5.6$ ). Furthermore, the students gave high ratings on the items that asked them to evaluate the ease, value, and use of this software as a learning tool. The students' responses resulted in a mean score of 5.4 on the item that asked to rate the quality of material presentation, and mean of 5.5 on the item that asked them to evaluate the worth of using this program as a tool to help in teaching the 
course. The results also point out that the quality of virtual reality system was acceptable to the required tasks, and that it has potential use as a tool for efficiently teaching students to perform virtual experiments and manipulate variables similar to real-world and hands-on experimentation activities.

Most of the students found the ability to adjust the system's parameters very useful. They enjoyed having a level of control over the system that is not possible in the real world, and they said it helped their understanding of the system. The real-time graph of the coupling efficiency was also a popular feature, as it allowed students to observe a trend in coupling efficiency rather than just a changing number. There were many students that wanted to do only the virtual lab module because they felt that it was easier and less time-consuming, but most of the students wanted to do both the virtual lab and the physical lab. In the future, we may explore making the module more difficult and expanding it to be more involved so that it may also be a better stand-alone product for an online course.

The conducted study clearly demonstrated the feasibility of the proposed instructional technology for engineering education, where both the model of instruction and the enabling technology are equally important in providing a better learning environment to improve students' conceptual understanding as compared to other instructional approaches. The module presented a simulation of a lab exercise that engaged the students with relatable visuals and easy-to-access controls. The students' survey responses suggest that they found the module interesting and would like to see similar labs implemented in optics courses in the future.

Acknowledgement: This research has been supported by US National Science Foundation grant EEC-0935006.

\section{REFERENCES}

[1] Brooks Jr., F., "What’s Real about Virtual Reality?" IEEE Computer Graphics and Applications 19(6), 16-27 (1999).

[2] van Veen, H., Distler, H. K., Braun, S. J. and Bulthoff, H. H., "Navigating through a virtual city: Using virtual reality technology to study human action and perception," Future Generation Computer Systems 14, 231-242 (1998).

[3] Dede, C., Salzman, M., Loftin, B and Ash, K., "Using Virtual Reality Technology to Convey Abstract Scientific Concepts," in M.J. Jacobson \& R.B. Kozma (Eds.), Learning the Sciences of the 21st Century: Research, Design, and Implementing Advanced Technology Learning Environments, Lawrence Erlbaum (1997).

[4] Balmush, N. and Dumbraveanu, R., "Virtual laboratory in optics," 3rd international conference on Multimedia and Information of Communication Technologies in Education, Caceres, Spain, June 7 - 10 $0^{\text {th }}(2005)$.

[5] Armstrong, S. and Mahmoud, A., "Experiential Learning and the Acquisition of Managerial Tacit Knowledge." s.l. : Academy of Management Learning \& Education 7(2), 189-208 (2008).

[6] Pompea, S., "Design of Computer-Assisted Education Programs for Optics and Photonics: Implications of Educational Technology Research," Proc. SPIE 4588, 414-422 (2002).

[7] Yair, Y., Mintz, R. and Litvak, S., "3D-Virtual Reality in Science Education: An Implication for Astronomy Teaching," Journal of Computers in Mathematics and Science Teaching 20(3), 293-305 (2001).

[8] Integra Web site, (October 13, 2009) www.integra.jp/en/specter/index.html.

[9] Zemax Software, (June 5, 2013) http://www.radiantzemax.com/zemax/.

[10] OptoDesigner, a light propagation simulator, (June 5, 2013) www.phoenixbv.com. 